\title{
Avaliação das Condições Higiênico-sanitárias de Serviços de Alimentação do Município de Unaí-MG: Antes e Depois
}

\section{Evaluation of the Sanitary Conditions of food Services of the City of Unaí-MG: Before and After}

\author{
Emiliane Veloso de Almeida Borges ${ }^{\mathrm{a}}$; Luciene Alves ${ }^{\mathrm{b}}$; Deborah Santesso Bonnas ${ }^{\mathrm{c}}$; Elisa Norberto Ferreira Santos ${ }^{\mathrm{c}}$; \\ Fernanda Barbosa Borges Jardim*c
}

\begin{abstract}
aSecretaria Municipal de Saúde, Departamento de Vigilância Sanitária. MG.
bUniversidade Federal do Triângulo Mineiro Departamento de Nutrição. MG.

'Instituto Federal de Educação, Ciência e Tecnologia do Triângulo Mineiro, Departamento de Pós-graduação em Ciência e Tecnologia de Alimentos. MG.

*E-mail: fernanda.jardim@iftm.edu.br.
\end{abstract}

\begin{abstract}
Resumo
Segurança do Alimento é um requisito obrigatório no segmento de serviço de alimentação, de forma a garantir a oferta de alimentos de qualidade e sem riscos à saúde dos consumidores. O objetivo do trabalho foi realizar o diagnóstico de estabelecimentos de serviços de alimentação em relação às Boas Práticas de Fabricação na cidade de Uná, MG e avaliar se as intervenções realizadas foram efetivas. Foi realizado um estudo descritivo de amostragem por conveniência. A metodologia consistiu nas seguintes etapas: a) palestra de sensibilização; b) aplicação de checklist inicial e relatório de diagnóstico inicial; c) plano de ação; d) treinamento sobre Boas Práticas; e) consultorias in loco; f) aplicação de checklist final e relatório de diagnóstico final; g) supervisão final e relatório de auditoria. Houve adesão de 13 empresas ao programa. Pôde-se observar que a média geral de conformidades no diagnóstico inicial foi de $57 \%$ e, após as intervenções e treinamento foi de $79 \%$. Houve uma diminuição de $23 \%$ das não conformidades nos estabelecimentos. Comprovou-se que as ações de intervenção foram significativas em dez empresas pelo teste exato de Fisher $(p<0,05)$. As maiores dificuldades encontradas, na maioria dos estabelecimentos, foram insuficientes controles essenciais durante a preparação e manutenção dos alimentos preparados e ausência de capacitações para os manipuladores de alimentos. Conclui-se que houve melhorias higiênico-sanitárias para todos os estabelecimentos e que as intervenções realizadas foram, em geral, efetivas para adequações às Boas Práticas.
\end{abstract}

Palavras-chave: Boas Práticas de Manipulação. Capacitação. Serviços de Alimentação.

\begin{abstract}
Food Safety is a mandatory requirement in the food service segment in order to ensure the quality food provision and without risks to the consumers' health. The objective of this study was to perform the diagnosis of food service establishments in relation to Good Manufacturing Practices in the city of Unai, $M G$, and to evaluate if the interventions were effective. A descriptive sampling study was conducted for convenience. The methodology consisted of the following steps: a) awareness-raising lecture; b) application of initial check list and initial diagnostic report; c) plan of action; d) training on good practices; e) on-site consultancy; f) application of final check list and final diagnostic report; g) final supervision and audit report. There were 13 companies joining the program. It was observed that the overall compliance mean at the initial diagnosis was $57 \%$ and after the interventions and training, it was $79 \%$. There was a 23\% decrease in non-conformities in the establishments. It was verified that the intervention actions were significant in ten companies by the Fisher's exact test $(p<0.05)$. The major difficulties encountered in most establishments were insufficient essential controls during the preparation and maintenance of prepared foods and lack of training for food handlers. It was concluded that there were hygienic-sanitary improvements for all the establishments and that the interventions performed were, in general, effective for adaptations to Good Practices.
\end{abstract}

Keywords: Good Manipulation Practices. Training. Food Services.

\section{Introdução}

As Boas Práticas de Fabricação (BPF) são compostas por um conjunto de princípios e regras para o correto manuseio dos alimentos, que abrangem desde a recepção das matériasprimas até o consumo final. O seu principal objetivo é garantir a integridade do alimento e a saúde do consumidor (FERRERIA et al., 2010). Em função de inexistência de Portarias e Normativas nacionais regulamentadoras das BPF em relação aos estabelecimentos fornecedores de alimentação, a Agência Nacional de Vigilância Sanitária - ANVISA publicou a RDC n² 216 de 15 de setembro de 2004 (RESOLUÇÃO RDC, 2004), que abrange os procedimentos que devem ser adotados nos serviços de alimentação, a fim de garantir as condições higiênico-sanitárias do alimento preparado. Essa legislação federal pode ser complementada pelos órgãos de vigilância sanitária estadual, distrital e municipal, visando abranger requisitos inerentes às realidades locais e promover a melhoria das condições higiênico-sanitárias dos serviços de alimentação (CUNHA; MAGALHÃES; BONNAS, 2012).

As Doenças Transmitidas por Alimentos - DTA são comuns em todos os países e têm se mostrado muito frequente não só em quantidade de surtos como na variedade de agentes etiológicos. Mesmo com todos os recursos técnicos disponíveis, como a implementação das BPF, os surtos têm aumentado nos países nos quais a cultura do povo é deficitária, o investimento empresarial é mínimo e o poder de fiscalização das Vigilâncias Sanitárias é restrito (ABERC, 2013). 
Regulamentar e fiscalizar são apenas alguns pontos, que podem contribuir para uma melhor qualidade dos alimentos preparados. Assim, convém que os gestores, nutricionistas e colaboradores dos serviços de alimentação sejam responsáveis por práticas e ambientes adequados para a manipulação de alimentos. Com o nível de informação restrito, os consumidores geralmente têm dificuldade em avaliar e mesmo cobrar dos estabelecimentos, que frequentam, uma postura rígida com relação aos aspectos sanitários. Por isso, o protagonismo dos empresários depende, principalmente, de um alto grau de consciência e de uma fiscalização atuante (MEDEIROS; PEREIRA; SACCOL, 2012).

A segurança do alimento é, portanto, o objetivo a ser alcançado no segmento de serviço de alimentação, de forma a garantir a oferta de alimentos de qualidade e sem riscos à saúde dos consumidores. O uso do termo alimento seguro é um conceito que está crescendo na conjuntura global, não somente pela sua importância para a saúde pública, mas também pelo seu importante papel no comércio internacional. Assim, a produção de alimentos e de refeições seguras envolve uma série de fatores, os quais merecem atenção no processo de gestão. Uma forma de demonstrar este intento é através do diagnóstico e adequação dos estabelecimentos, quando se aplicam as BPF (CUNHA; MAGALHÃES; BONNAS, 2013).

A cidade de Unaí, MG, de acordo com o censo de 2016, do Instituto Brasileiro de Geografia e Estatística (IBGE, 2016), possui uma população estimada em 83.448 pessoas. O setor de serviços de alimentação de micro e pequenas empresas da cidade possui apoio do Serviço Brasileiro de Apoio às Micro e Pequenas Empresas - SEBRAE e Serviço Nacional de Aprendizagem Comercial - SENAC para promover a competitividade e o desenvolvimento sustentável dos pequenos negócios e fomentar o empreendedorismo para fortalecer a economia local.

O objetivo da pesquisa foi avaliar as BPF de 13 empresas de serviços de alimentação na cidade de Unaí, MG, e verificar se as intervenções realizadas foram efetivas para adequações dos estabelecimentos.

\section{Material e Métodos}

Foi realizado um estudo descritivo de amostragem por conveniência realizado em parceria com o SEBRAE de Unaí, MG, e SENAC sediado em Belo Horizonte, MG. A pesquisadora, consultora do Programa de Alimentos Seguros do SENAC, Uberlândia, MG, foi executora da avaliação e adequação de estabelecimento quanto às BPF.

A metodologia envolveu as etapas iniciais de sensibilização de estabelecimentos de Serviços de Alimentação com sede em Unaí, MG, para adequação das BPF e adesão das empresas para a primeira etapa do projeto, que consistiu no diagnóstico inicial das conformidades e não conformidades e relatório fotográfico dos estabelecimentos. Participaram das etapas iniciais 30 estabelecimentos, sendo 13 restaurantes comerciais, seis açougues, cinco panificadoras, quatro cozinhas de hotel, um supermercado e uma sorveteria. Houve adesão de 13 empresas, que se mostraram interessadas em dar continuidade ao programa: nove restaurantes comerciais, duas panificadoras, uma cozinha de hotel e uma sorveteria. $\mathrm{O}$ processo de implantação de BPF seguiu um fluxo representado pela Figura 1.

Figura 1 - Fluxograma geral de aplicação de Boas Práticas de Fabricação em estabelecimentos de serviços de alimentação

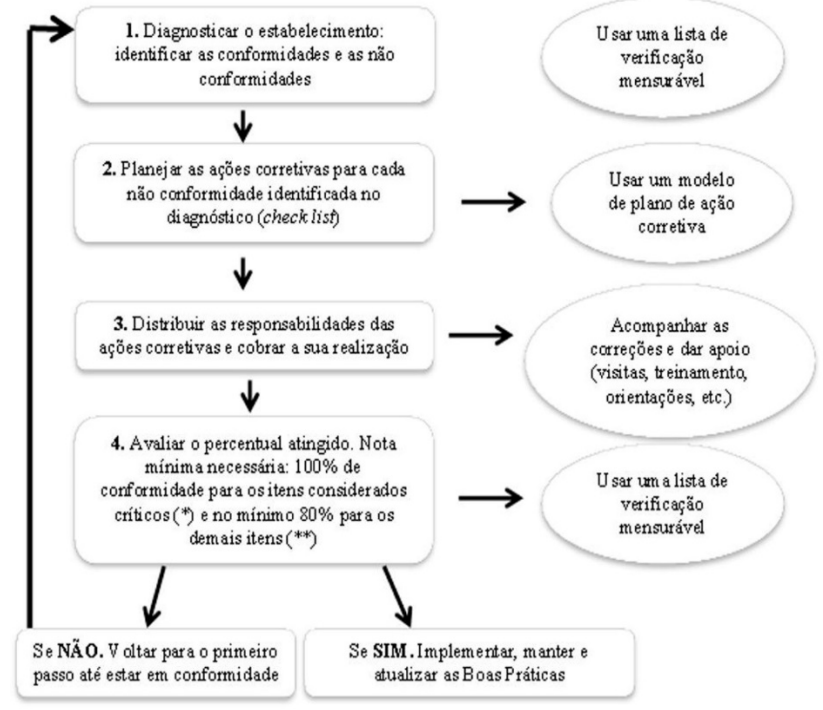

Fonte: Adaptada de Bertin e Mendes ${ }^{8}$.

No diagnóstico inicial foi efetuado relatório fotográfico e aplicado o checklist baseado na RDC 216/2004 (RESOLUÇÃO RDC, 2004), sem categorização dos estabelecimentos, contemplando os seguintes aspectos: edificação, instalações, equipamentos, móveis e utensílios; higienização de instalações, equipamentos, móveis e utensílios; controle integrado de vetores e pragas urbanas; abastecimento de água; manejo de resíduos; manipuladores; matérias-primas, ingredientes e embalagens; preparação do alimento; armazenamento e transporte do alimento preparado; exposição ao consumo do alimento preparado; documentação e registro; responsabilidade técnica.

Os itens analisados foram classificados da seguinte forma: Conforme (C): itens que estão de acordo com a legislação; Não conforme (NC): itens que não estão de acordo com a legislação; Não aplicável (NA): itens que não estão relacionados com as atividades exercidas pela empresa; Não observado (NO): itens que não foram observados durante a visita.

As atividades de adequação dos estabelecimentos em BPF seguiram as seguintes etapas: plano de ação entregue às empresas (relatório inicial); treinamento sobre BP (Boas Práticas); esclarecimento de dúvidas e orientações in loco para adequação dos estabelecimentos; aplicação de checklist final e elaboração de relatório final; supervisão final e relatório de auditoria.

O treinamento sobre BPF foi realizado com carga horária 
total de 20 horas, seguindo metodologia de exposição dialogada e com utilização de recursos audiovisuais. A duração do curso foi de cinco dias e participaram duas turmas em cada período, com carga horária diária de 4 horas. O conteúdo programático do treinamento foi (RESOLUÇÃO RDC, 2004, SENAI, 2012; SENAC, 2012):

- Legislação Sanitária / Noções de Microbiologia de Alimentos / Perigo nos Alimentos / Doenças de Origem Alimentar (DOA)

- Normas para Estrutura Física das Unidades de Produção e Serviços de Alimentação

- Controle de Água / Controle de Vetores e Pragas Urbanas / Manejo de Resíduos

- Cuidados com os Manipuladores de Alimentos / A importância do uso do EPI (Equipamento de Proteção Individual)

- Boas Práticas de Fabricação: Critérios de Segurança na produção e manipulação de alimentos

No total, participaram 76 colaboradores no treinamento, sendo exigida frequência mínima de $75 \%$ para emissão do certificado. Todos os participantes receberam uma cartilha de orientação para manipuladores de alimentos (SENAI, 2012); e os empresários receberam um kit de cartilhas para manipuladores de alimentos, materiais padronizados e elaborados pelo SENAC, MG (SENAC, 2012). Ao término do curso, todos os colaboradores frequentes receberam um certificado de conclusão de curso emitido pelo SEBRAE de Unaí.

As avaliações iniciais e finais dos estabelecimentos foram tabuladas e tratadas, estatisticamente, para obtenção de panorama geral de evolução das condições higiênicosanitárias dos estabelecimentos de serviços de alimentação da cidade de Unaí, MG. A análise estatística utilizada foi o Teste exato de Fisher. Os itens avaliados nas empresas, não conformes e conformes, foram comparados individualmente contra uma variável resposta (análise univariada) através de tabelas de contingência do tipo dois por dois $(2 \times 2)$. A avaliação estatística analisou se a variável de linha e a variável de coluna são independentes, considerando a hipótese $\mathrm{H}_{0}$ de que não existiu diferença estatística após a intervenção nas empresas; e a hipótese $\mathrm{H}_{1}$, de que existiu diferença estatística após a intervenção nas empresas. A significância estatística foi definida para um valor de $\mathrm{p}$ menor que $0,05(p<0,05)$. A análise das variáveis foi realizada sendo utilizado o programa estatístico GraphPad Prism4.

Todas as ações de implantação de BPF, inclusive o treinamento, foram analisadas com apresentação das principais dificuldades enfrentadas durante o processo e os pontos positivos das atividades. A elaboração do relatório e supervisão final foram realizadas por um consultor/auditor externo encaminhado pelo SENAC (Unidade Belo Horizonte) com o objetivo de validar o processo de implantação das BPF nos estabelecimentos e auditar as ações da pesquisadora, executora do projeto.

\section{Resultados e Discussão}

Os resultados obtidos, a partir da aplicação do checklist inicial e final das 13 empresas de serviços de alimentação, que aderiram ao programa, podem ser visualizados no Quadro 1.

Quadro 1 - Resultados do checklist baseado na RDC 216/2004² inicial e final aplicado em 13 estabelecimentos de serviços de alimentação do município de Unaí, MG, segundo Boas Práticas de Fabricação

\begin{tabular}{|l|c|c|c|c|}
\hline Checklist & $\begin{array}{c}\text { Itens } \\
\text { Conformes }\end{array}$ & $\begin{array}{c}\text { Itens não } \\
\text { Conformes }\end{array}$ & $\begin{array}{c}\text { Itens não } \\
\text { Observados }\end{array}$ & $\begin{array}{c}\text { Itens não } \\
\text { Aplicáveis }\end{array}$ \\
\hline Inicial & $57 \%$ & $38 \%$ & $1 \%$ & $3 \%$ \\
\hline Final & $79 \%$ & $15 \%$ & $0 \%$ & $6 \%$ \\
\hline
\end{tabular}

Fonte: Dados da pesquisa.

Observa-se aumento médio de $22 \%$ de conformidades nos 13 estabelecimentos analisados e diminuição de $23 \%$ das não conformidades comparando-se os resultados das avaliações inicial e final (Quadro 1).

A maior dificuldade encontrada durante a consultoria, na maioria dos estabelecimentos, foi a ausência de controles essenciais, como controle de temperatura na preparação e distribuição e higiene dos alimentos, além da não realização de capacitações para os manipuladores.

Das empresas participantes, 70\% não possuíam Manual de BPF, Procedimentos Operacionais Padronizados, planilhas de controle de temperaturas, planilhas e cronograma de manutenções e higienização de equipamentos/instalações, laudos de prestação de serviço de controle de vetores e pragas urbanas e laudos de limpeza da caixa de água. Assis (2011) ressalta que os registros funcionam como evidências de atividades realizadas e seus resultados. Isso confere credibilidade e demonstra a eficácia e a eficiência de ferramentas implementadas pela empresa.

Santos e Pinto (2015) avaliaram as BPF, em restaurantes de autosserviço, em São Paulo e identificaram que a falta de documentação era presente em $80 \%$ dos restaurantes. As autoras observaram que essa falha ocorria concomitante à falta de um responsável técnico.

Quanto aos manipuladores de alimentos, 77\% dos estabelecimentos não apresentaram atestados de saúde dos colaboradores, que evidenciem a aptidão nas atividades exercidas e registros de capacitações, que comprovem as habilidades dos colaboradores, em relação aos temas mínimos estabelecidos pela RDC 216/04: Contaminantes alimentares; Doenças transmitidas por alimentos; Manipulação higiênica dos alimentos e Boas Práticas.

Houve deficiência, por parte dos responsáveis e dos colaboradores, quanto à existência e aplicabilidade das legislações vigentes, que envolvem as BPF para Serviços de Alimentação. Por não ter nenhum funcionário ou proprietário devidamente capacitado em cursos para a manipulação de alimentos se torna mais difícil a implantação, uma vez que é essencial que toda a equipe esteja bem sincronizada e capacitada para o desenvolvimento e aperfeiçoamento 
das ações de segurança estabelecidas na empresa. Essas dificuldades de conhecimento das legislações de BPF, juntamente com a consultoria, fizeram com que proprietários e colaboradores trabalhassem, mutuamente, em prol de melhorias para os estabelecimentos.

Nesse contexto, a realização do treinamento de BPF foi essencial para aumentar o nível de entendimento dos proprietários e colaboradores, facilitando o trabalho de consultoria. Por meio da capacitação, foi possível apresentar ferramentas para garantir a qualidade e segurança dos alimentos preparados e comercializados nos estabelecimentos de serviços de alimentação participantes.

Pittelkow e Bitello (2014) avaliaram a higiene pessoal e o comportamento dos manipuladores de alimentos de uma Unidade de Alimentação e Nutrição (UAN) com melhora dos itens conformes de $61,11 \%$ (antes do treinamento) para $72,22 \%$ (após o treinamento). O índice de melhora foi inferior $(11,11 \%)$ ao encontrado no presente estudo, que obteve aumento de $22 \%$ nos índices de conformidades.

Centurione e Santos (2016) constataram que, no setor de alimentação, há alta rotatividade de mão de obra e que são contratados trabalhadores sem experiência, tornando-se necessária a capacitação dos mesmos durante as atividades e no momento da contratação. No presente estudo, 78,9\% dos manipuladores não receberam treinamento quando foram contratados e $52,6 \%$ não fizeram curso de manipulador de alimentos antes da contratação. Entre os que realizaram curso para manipulador de alimentos, o período de duração foi de apenas três horas. Segundo a RDC 216/2004, não é especificada a carga horária mínima dos cursos de capacitação, mas o conteúdo programático deve abordar, no mínimo, os temas contaminantes alimentares; doenças transmitidas por alimentos; manipulação higiênica dos alimentos e Boas Práticas.

Medidas preventivas a serem tomadas junto aos manipuladores de alimentos são fundamentais e a melhor forma de fornecer conhecimento a eles é a informação. O curso de capacitação para manipuladores de alimentos resultou em benefícios para as empresas quanto ao controle higiênico-sanitário de seus produtos e processos, uma vez que houve a possibilidade de desenvolver ações capazes de eliminar, diminuir ou prevenir os riscos.

Durante e após a realização do treinamento e com as visitas orientativas in loco, as empresas demonstraram interesse em melhorar as condições estruturais, de manutenção, de organização e de limpeza. Vale ressaltar a grande relevância da participação das autoridades sanitárias nesse processo, pois além da implantação das BPF, por parte da empresa, é fundamental que a implementação seja validada pelos órgãos sanitários fiscalizadores.

Um total de 10 empresas apresentaram melhorias significativas $(p<0,05)$ após as adequações em BPF e três empresas não apresentaram melhorias.

Uma empresa, classificada como cozinha de hotel, não obteve valores de melhorias significantes, provavelmente, pelo fato de sua atividade, no âmbito de aplicação, ser apenas de fornecimento de alimentos prontos para consumo (há uma cozinha terceirizada que manipula, prepara e entrega somente o café da manhã é servido neste local). Portanto, não houve grandes mudanças em seu processo.

As empresas que não conseguiram se adequar quanto às $\mathrm{BPF}$ relacionadas às melhorias estruturais e contratação de mão de obra especializada relataram dificuldades financeiras.

Os resultados atestaram que a consultoria, em geral, foi eficiente e resultou em melhoria das condições higiênicosanitárias de aproximadamente $77 \%$ dos estabelecimentos de serviços de alimentação da cidade de Unaí, MG.

A partir das não conformidades citadas se constatou que o sistema de gestão de qualidade deve ser administrado como responsabilidade primária e que precisa ser monitorado, vistoriado e levado ao nível seguro para quem consome. O crescimento destes estabelecimentos demonstra que é inevitável o constante aprimoramento das normas de qualidade, bem como de um serviço de gestão de qualidade eficiente, em que o manipulador é o responsável direto pelo produto final a ser consumido e deve receber treinamento e revisão de atualização constantemente.

Os estabelecimentos foram informados das inúmeras vantagens das BPF, a saber: econômicas com redução de custo através do melhor conhecimento dos seus processos; redução de desperdícios de matérias-primas e insumos; agregação de valor ao produto com a inserção do fator qualidade; aumento da produtividade; capacitação e motivação do manipulador; ações que contribuem para um produto final de melhor qualidade e seguro para o consumo.

Garcia e Centenaro (2016) destacam em estudo que o aumento do nível de adequação de um serviço de alimentação foi auxiliado pelo preenchimento do Plano de Ação. A elaboração do modelo de plano de ação foi sugerido, pois a ANVISA indica esse método para planejar as adequações necessárias contidas no Regulamento Técnico de Boas Práticas para Serviços de Alimentação.

Um mês após a conclusão do relatório de diagnóstico final, foi realizada a supervisão através de uma visita técnica, realizada por um consultor/auditor externo encaminhado pelo SENAC (Unidade de Bolo Horizonte, MG) na qual foram efetuadas as avaliações que incluem observações "in loco" da estrutura física, dos procedimentos de BPF aplicados na manipulação e da análise da documentação e registros.

A auditoria apresentou um relatório de supervisão de análise de documentos, pontos fortes, oportunidades de melhoria e parecer técnico, auditando as ações da pesquisadora e validando, positivamente, o processo de implantação das BPF nos estabelecimentos. Da análise realizada "in loco" foi possível destacar alguns pontos das empresas que apresentaram relevância no cumprimento de metas para implantação das BPF (Quadro 2). 
Quadro 2 - Análise de Pontos Fortes e Oportunidades de Melhorias descritas em Relatório de Supervisão Final das empresas que apresentaram relevância no cumprimento de metas para implantação das Boas Práticas de Fabricação $(\mathrm{n}=10)$

\section{Melhorias Estruturais (Pontos Fortes Analisados)}

- Capacitação de parte da equipe em Boas Práticas;

- Separação de lavatório exclusivo para higienização das mãos com todas as facilidades e cartazes informativos;

- Adequação e organização da estrutura física com a divisão do espaço para melhorar o fluxo de produção e separação das áreas;

- Instalação de telas milimétricas removíveis em todas as aberturas para áreas externas;

- Armazenamento de alimentos em temperatura adequada, porcionamento e identificação;

- Foram encontradas as instalações, equipamentos e utensílios em condições higiênicas e sanitárias adequadas;

- Aquisição de lixeiras com acionamento por pedal para todas as áreas;

- Realizado controle integrado de vetores e pragas, com empresa especializada;

- Higienização do reservatório de água e registro;

- Instalação de proteção contra quebra e explosão nas luminárias sobre a área de preparo dos alimentos.

\section{Oportunidades de melhorias}

- Monitorar e registrar a temperatura durante a distribuição nos balcões de serviço quente e frio, registrando e corrigindo as eventuais falhas;

- Monitorar a temperatura das matérias-primas refrigeradas na recepção, assim como dos equipamentos, registrando e corrigindo as eventuais falhas;

- Adequação do procedimento para higienização de frutas e verduras, utilizando solução a $200 \mathrm{mg} \mathrm{L}^{-1}$ de hipoclorito de sódio ou outro produto recomendado, encontrado em casas especializadas;

- Elaboração do Manual de Boas Práticas e os quatros Procedimentos Operacionais Padronizados.

Fonte: Dados da pesquisa.

A supervisão final emitiu o parecer técnico no qual consta que a direção da empresa valorizou e se apoiou nas BPF para segurança dos alimentos comercializados no seu estabelecimento. O comprometimento da administração é um dos requisitos que garante o sucesso do programa (Quadro 2).

As empresas que não obtiveram avanços significativos, pelo fato de não apresentarem melhorias em relação ao checklist apresentado e cumprimento de metas do plano de ação, foram analisadas conforme descrição no Quadro 3.

Quadro 3 - Análise de Pontos Fortes e Oportunidade de Melhorias descritas em Relatório de Supervisão Final das empresas que não apresentaram relevância no cumprimento de metas para implantação das Boas Práticas de Fabricação $(\mathrm{n}=3)$

\section{Melhorias Estruturais (Pontos Fortes Analisados)}

- Capacitação de parte da equipe em curso de Boas Práticas de Fabricação;

- Adequação e organização da estrutura física com instalação de telas milimétricas removíveis em janelas e portas, mas falta em exaustores;

- Reorganização da área de estoque com separação de alimentos, embalagens e material de higienização;

- Separação de equipamento de refrigeração para tipos diferentes de alimentos;

- Construção de sanitários para colaboradores com disponibilização de materiais de higiene;

- Foram encontradas as instalações, equipamentos e utensílios em condições higiênicas e sanitárias adequadas;

\section{Oportunidades de melhorias}

- Instalar proteção contra quebra e explosão das lâmpadas na área de processamento;

- Contratar empresa especializada para o controle de pragas e vetores urbanos;

- Realizar a higienização do reservatório de água a cada seis meses e registrar a data do procedimento;

- Manter o lavatório já presente na produção para a higienização das mãos com todas as facilidades, como: sabonete antisséptico, ou sabonete e produto antisséptico e fixar cartaz informativo e monitoramento;

- Monitorar a temperatura das matérias-primas refrigeradas na recepção, assim como dos equipamentos de refrigeração, registrando e corrigindo as eventuais falhas;

- Monitorar e registrar as temperaturas dos alimentos na exposição nos balcões quentes e frios, registrando e corrigindo as eventuais falhas.

- Confeccionar e distribuir uniformes e EPI completos para os colaboradores, assim como monitorar sua troca diária. Verificar a retirada pelos colaboradores de todos os adornos e manutenção de unhas curtas e sem esmalte;

- Encaminhar colaboradores para exames laboratoriais de comprovação da saúde;

- Separar um equipamento de refrigeração para descongelamento das carnes, evitando assim exposição em temperaturas de zona de perigo para crescimento de micro-organismos;

- Fazer manutenções periódicas nos equipamentos de refrigeração, pois alguns não estão mantendo a temperatura, além de desperdiçarem muita energia elétrica;

- Trocar os equipamentos que têm madeira na estrutura, pois podem ser abrigos de pragas.

- Elaboração do Manual de Boas Práticas de Fabricação e os quatros Procedimentos Operacionais Padronizados.

Fonte: Dados da pesquisa.

A supervisão final emitiu o parecer técnico em que consta que a direção da empresa não pôde se empenhar muito para a implantação das BPF, mas mesmo assim houve melhorias. Faltou implementação para maior evolução, o que é imprescindível para que seja bem-sucedida a implantação. O comprometimento da administração é o principal requisito que garante o sucesso do programa. Ficou como orientação que o proprietário e funcionários devem seguir o plano de ação já descrito pela consultora para cada não conformidade pendente e identificada durante a supervisão (Quadro 3).

Uma das formas para se atingir a segurança alimentar do ponto de vista higiênico-sanitário é mediante a implementação do programa de BPF (COELHO; TOLEDO, 2017). A principal meta das BPF é a máxima redução dos riscos e o aumento da qualidade e da segurança dos alimentos.

\section{Conclusão}

Houve avanço nas adequações higiênico-sanitárias nos estabelecimentos participantes do seguimento alimentício da cidade de Unaí-MG e as intervenções realizadas foram efetivas para implantação de BPF. Dos 13 estabelecimentos, que participaram do estudo, 10 serviços de alimentação (77\%) apresentaram melhora significativa, entretanto todos aumentaram os índices de conformidade.

Para que o alimento servido seja de boa qualidade e com riscos controlados, é fundamental que os estabelecimentos de 
serviços de alimentação de Unaí-MG deem continuidade às ações implantadas e implementadas.

\section{Agradecimentos}

Ao SEBRAE (Serviços Brasileiro de Apoio às Micro e Pequenas Empresas) de Unaí - MG, SENAC (Serviço Nacional de Aprendizagem Comercial) e IFTM (Instituto Federal de Educação, Ciência e Tecnologia do Triângulo Mineiro) pelo apoio técnico na realização da pesquisa.

\section{Referências}

ABERC - Associação Brasileira das Empresas de Refeições Coletivas. Manual Aberc de práticas de elaboração e serviços de refeições para coletividades. São Paulo: ABERC, 2013.

ASSIS L. Alimentos seguros: ferramentas para gestão e controle da produção e distribuição. Rio de Janeiro: Senac Nacional, 2011.

CENTURIONE, L.C.F.; SANTOS, P.L.R. Avaliação da segurança alimentar dos serviços de alimentação permissionários dos campi de uma Universidade Federal. Niterói: Universidade Federal Fluminense, 2016.

COELHO, R.P.; TOLEDO, J.C. Programas para segurança na indústria de alimentos para animais: caracterização e benefícios percebidos com a implantação. Gest. Prod., v.24, n.4, p.704-718, 2017. doi: 10.1590/0104-530x2262-16.

CUNHA, F.M.F.; MAGALHÃES, M.B.H.; BONNAS, D.S. Desafios da gestão da segurança dos alimentos em unidades de alimentação e nutrição no Brasil: uma revisão. Contextos Aliment., v.1, n.2, p.4-12, 2012.

CUNHA, F.M.F.; MAGALHÃES, M.B.H.; BONNAS, D.S. Desafios da gestão da segurança dos alimentos em unidades de alimentação e nutrição no Brasil: uma revisão. Contextos Aliment., v.2, n.2, p.4-14, 2013.

FERREIRA, A.A. et al. Dificuldades de implantação do sistema da qualidade de pequenas e médias empresas alimentícias. Rev. Unilins, 2010. Disponível em: <http://revista.unilins.edu.br/ index.php/cognitio/article/view/7/9>. Acesso em: 30 fev. 2018.

GARCIA, M.V.; CENTENARO, G.S. Capacitação de manipuladores de alimentos e avaliação das condições higiênicas em serviço de alimentação. Braz J. Food. Technol., v.7, n.2, p.96111, 2016. doi: 10.14685/rebrapa.v7i2.3640.

IBGE - Instituto Brasileiro de Geografia e Estatística. Minas Gerais Censo Unaí 2016. Disponível em: <https://cidades.ibge. gov.br/brasil/mg/unai> Acesso em: 17 maio 2019.

MEDEIROS, L.B.; PEREIRA, L.C.; SACCOL, A.L.F. Atitudes de risco dos consumidores em self-service. Rev. Inst. Adolfo Lutz, v.71, n.4, p.737-740, 2012.

PITTELKOW, A; BITELLO RAA. Higienização de manipuladores de uma Unidade de Alimentação e Nutrição (UAN). Rev. Destaques Acadêm., v.6, n.3, p.22-27, 2014.

RESOLUÇÃO RDC n 216. Dispõe sobre o Regulamento Técnico de Boas Práticas para Serviços de Alimentação 2004 set. 15. Pub DO.

SANTOS, D.F.S.; PINTO, A.T.B. Avaliação das boas práticas de fabricação em restaurantes self-service da cidade de Itapeva, estado de São Paulo. Rev. Nutr., v.1, n.4, p.1-9, 2015.

SENAC. Serviço Nacional de Aprendizagem Comercial. Segurança na manipulação dos alimentos: missão (super) impossível. Rio de Janeiro: SENAC Nacional, 2012.

SENAI. Serviço Nacional de Aprendizagem Industrial. Cartilha do manipulador de alimentos: mesa. Brasília: SENAI, 2009. 